\title{
NA TEIA DA FICCÃO: A COMPLEXA NARRATIVA DA SÍNDROME DE MUNCHAUSEN POR PROCURAÇÃO
}

\author{
Elisabete Lopes \\ Doutoranda em Estudos da Criança - CIEC, Instituto de Educação, Universidade do Minho, Braga, Portugal \\ id9952@alunos.uminho.pt \\ Zélia Caçador Anastácio \\ CIEC, Instituto de Educação, Universidade do Minho, Braga, Portugal, zeliaf@ie.uminho.pt
}

Recepción Artículo: 13 octubre 2021 Admisión Evaluación: 13 octubre 2021 Informe Evaluador 1: 13 octubre 2021 Informe Evaluador 2: 14 octubre 2021 Aprobación Publicación: 14 octubre 2021

\section{RESUMO}

A Síndrome de Munchausen por Procuração, também designada como Transtorno Factício Imposto a Outro, é definida como uma perturbação significativa, complexa e, por conseguinte, de difícil controlo e diagnóstico. A sua complexidade deve-se ao facto de ser considerada como tipologia de abuso infantil em que 0 agressor, geralmente a mãe, induz ou simula, deliberadamente, sinais e/ou sintomas de doença no filho/a com intuito de obter atenção para si. É representada por uma relação triangular que envolve, mãe-agressor, filho/a-vítima e médicoprofissional de saúde, sendo este último utilizado pelo/a perpetrador/a do abuso para satisfação da sua necessidade intrínseca e, por consequência, na perpetuação do abuso. 0 presente artigo propõe uma revisão narrativa da literatura sobre a Síndrome de Munchausen por Procuração tentando enquadrá-la à luz da designação do abuso infantil, ao mesmo tempo que procura alertar para a necessidade da sua identificação precoce pelos profissionais de saúde, visto que este comportamento pode conduzir à morte da criança.

Palavras-chave: síndrome de Munchausen por procuração; transtorno factício imposto a outro; abuso infantil

\section{ABSTRACT}

In the web of fiction: the complex narrative of Munchausen Syndrome by Proxy.

The Munchausen Syndrome by Proxy, also known as Factitious Disorder Imposed on Another, is defined as a significant, complex and therefore difficult to control and diagnose disorder. Its complexity is due to the fact that it is considered as a type of child abuse in which the aggressor, usually the mother, deliberately induces or simulates signs and/or symptoms of illness in the child in order to obtain attention for him/herself. It is represented by a triangular relationship that involves mother-aggressor, son/daughter-victim and doctor-health professional, the latter being used by the perpetrator of the abuse to satisfy his or her intrinsic need and, 


\section{NA TEIA DA FICÇÃO: A COMPLEXA NARRATIVA DA SÍNDROME DE MUNCHAUSEN POR PROCURAÇÃo}

consequently, in the abuse perpetuation. The present article proposes a narrative review of the literature on Munchausen Syndrome by Proxy trying to fit it in the light of the designation of child abuse, emphasizing the behavior of the aggressor and the victim, at the same time that it seeks to alert to the need for its early identification by health professionals, as this behavior can lead to the child's death.

Keywords: Munchausen syndrome by proxy; factitious disorder imposed on another; child abuse.

\section{INTRODUÇÃO}

Munchausen faz alusão à personagem do século XVIII, Barão de Munchausen, que contava histórias dos seus atos heroicos de forma exagerada (Criddle, 2010; Franco et al., 2020), tendo ficado conhecido como 0 "barão das mentiras" (Thomas, 2003; Olry \& Haines, 2006; Garrote et al., 2008; Alicandri-Ciufelli et al., 2012; cit in Cascais, 2012, p.8.) e usado como inspiração para a denominação da síndrome (Silva \& Priszkulnik, 2013). Asher usou pela primeira vez o termo Síndrome de Munchausen, em 1951 (Filho et al., 2017). 0 autor nomeou esta patologia para descrever indivíduos que falsificavam histórias de doenças de forma recorrente e dramática (Ferrão \& Neves, 2013) com 0 intuito de se tornarem pacientes e serem submetidos a tratamentos médicos que se configuravam como supérfluos e inúteis (Franco et al., 2020; Criddle, 2010; Algeri et al., 2014).

Por sua vez, em 1977, Meadow identifica e edifica a Síndrome de Munchausen por Procuração na pediatria (Franco et al., 2020). Esta é traçada e interpretada como um quadro "severo e especial de abuso infantil" (Comert et al., 2018, p. 86) que consiste numa indução ou simulação deliberada de sinais e sintomas de doença na criança por parte do progenitor ou cuidador, tendo como finalidade a obtenção de atenção, simpatia e compaixão para si (Franco et al., 2020; Ferrão \& Neves, 2013; Pires \& Molle, 1999; Filho et al., 2017; Silva \& Priszkulnik, 2013). Neste sentido, a Sociedade Profissional Americana sobre o Abuso de Crianças "usa a designação falsificação de condição pediátrica (para a criança) e transtorno factício por procuração (para o perpetuador)" (Criddle, 2010, p.47). A Síndrome de Munchausen por Procuração é entendida, por alguns autores como "uma estranha combinação entre abuso físico, negligência médica e maus tratos psicológicos que ocorrem com o envolvimento ativo da profissão médica" (Donald \& Jureidini cit in Criddle, 2010, p. 47). Por outro lado, e porque esta síndrome se representa por uma relação triangular, Roesler e Jenny formaram a propósito a nomenclatura "abuso médico infantil" (Greiner et al., 2015, p. 39) para designar qualquer dano provocado na criança em virtude das ações médicas instigadas pelos relatos do portador da patologia que pode ser a mãe ou cuidador (Greiner et al., 2015). Visando 0 exposto, esta patologia é altamente complexa e é igualmente considerada uma tipologia de abuso infantil, pois o comportamento do portador desta condição pode conduzir à morte da criança. Por esta razão, com este trabalho pretendemos apresentar uma revisão narrativa da literatura que nos permita adquirir conhecimento atualizado sobre esta síndrome, enquadrá-la na tipologia de abuso infantil e alertar para a necessidade de os profissionais de saúde obterem precocemente 0 diagnóstico e assim identificarem os seus portadores.

\section{DEFINIÇÃO E DIAGNÓSTICO}

As patologias mencionadas encontram-se descritas na Classificação Internacional de Doenças (CID-10) e no Manual de Diagnóstico e Estatístico de Transtornos Mentais (DSM-V). Por esse motivo impõe-se definir "transtorno mental" para melhor se compreender as descrições das síndromes. Segundo o DSM-V, qualquer transtorno identificado no manual deve cumprir a definição de transtorno mental, que é entendida como "(...) síndrome caracterizada por perturbação clinicamente significativa na cognição, na regulação emocional ou no comportamento de um indivíduo que reflete uma disfunção nos processos psicológicos, biológicos ou de desenvolvimento associados a sofrimento ou incapacidade significativos que afetam atividades sociais, profissionais ou outras atividades importantes" (DSM-V, 2013, p.20).

A Síndrome de Munchausen (SM) faz referência ao facto de o próprio portador da patologia se colocar deliberadamente na condição de doente, enquanto que na Síndrome de Munchausen por Procuração (SMP) é o portador da patologia que coloca intencionalmente a condição de doente num outro indivíduo (Tachibana \& Ferreira, 2020), de forma deliberada (Franco et al., 2020) e premeditada, sendo este outro "(...) um idoso, uma pessoa 
com deficiência e (...) um animal, geralmente é uma criança em idade pré-escolar" (Braga; Saad cit in Tachibana \& Ferreira, 2020, p. 231).

A primeira condição (SM) encontra-se definida no CID-10, mais concretamente no capítulo que faz alusão aos Transtornos mentais e comportamentais, enquadrado nos Transtornos da personalidade e do comportamento adulto, sob a categoria F68.1 - Produção intencional ou simulação de sintomas ou deficiências, físicas ou psicológicas (Transtorno Factício), enquanto a segunda condição (SMP) se encontra prevista na categoria T74.8 Outras síndromes específicas de maus tratos (CID-10, 2019; Franco et al., 2020). Por outro lado, no DSM-V, as patologias encontram-se inseridas no capítulo Transtorno de Sintomas Somáticos e Transtornos Relacionados, onde se inclui o Transtorno Factício Autoimposto (Síndrome de Munchausen) e o Transtorno Factício Imposto a Outro (Síndrome de Munchausen por Procuração), onde os critérios de diagnóstico (para ambas) explanam a falsificação e indução de doença associada a fraude identificada, o comportamento ludibrioso, sendo evidente mesmo na carência de recompensas, e a compreensão do comportamento que não se enquadra ou se explica por outros transtornos, deferindo na condição de outro, em virtude de na Síndrome de Munchausen por Procuração, ser no outro que a doença se manifesta e sendo este outro apresentado como doente (DSM-V, 2013; Franco et al., 2020), admitindo que "a característica essencial do transtorno factício é a falsificação de sinais e sintomas médicos ou psicológicos em si mesmo ou em outro associado a fraude identificada" (DSM-V, 2014, p. 326).

A condição clínica é, portanto, uma forma de abuso infantil que consiste na fabricação ou indução voluntária de sinais e sintomas de doença na criança, com recurso à mentira, onde os factos são narrados como se fossem verdade (Zenoni, 2002). É imperativo afirmar que esta "condição psicopatológica" (Tachibana \& Ferreira, 2020, p.230) está correlacionada à violência exercida sobre crianças e a situações que se afiguram de perigo.

\section{CARACTERÍSTICAS, PERFIL E RELAÇÃO TRIANGULAR}

A literatura aponta 0 agressor como sendo, em cerca de 90\% dos casos, a mãe (Pires \& Molle, 1999; Silva \& Priszkulnik, 2013; Franco et al., 2020; Cascais, 2012). Bass e Glaser (cit in Telles et al., 2015) referem que 0 conceito de indução e simulação de doença vai desde negligência extrema até à morte da criança, havendo estudos que apontam os $9 \%$ (Marcus cit in Pires \& Molle, 1999) e outros que referem uma variação entre $6 \%$ a $10 \%$ de mortalidade (Anderson, Feldman \& Bryce cit in Tachibana \& Ferreira, 2020; Filho et al., 2017; Telles et al., 2015), configurando-se assim numa violência letal. "Para dar veracidade aos sintomas descritos, a criança pode ser vítima de episódios de sufocamento, envenenamento ou lesões (...)" (Emison \& Postlethwaite cit in Algeri et al., 2014, p. 3984). Os métodos mais comuns de indução de doença explanados na literatura encontram-se definidos em quatro categorias, nomeadamente 1) envenenamento - através do uso de substâncias como laxantes, sal; 2) hemorragias - através de uma variedade de métodos e podem ser simuladas ou produzidas, por exemplo hemorragia gastrointestinal; 3) infeções - podem resultar por aplicação de material fecal e urina e 4) lesões estas não sugerem imediatamente abuso físico, contudo são provocadas de forma intencional e podem ter como resultado fraturas, conjuntivites (Criddle, 2010; Cascais, 2012), sendo a primeira a forma mais descrita, cerca de $37 \%$ (Algeri et al., 2014) começando em casa com pequenas porções, escalando ao ponto de haver necessidade de hospitalização (Pires \& Molle, 1999).

Por outro lado e segundo Franco (et al., 2020, p.54), os autores definem sinais e sintomas que motivam desconfiança para uma possível situação de Síndrome de Munchausen por Procuração. Aqui denunciam e sublevam o desejo em se submeterem a procedimentos invasivos, história clínica vasta e de várias áreas de especialização, incluindo a existência de cirurgias, a resistência em facultar acesso a outras fontes de informação, como por exemplo a outros familiares, enquanto que outros autores apontam dois fatores como determinantes, nomeadamente crianças que apenas manifestam sinais de doença na presença da mãe ou apresentam, quando esta se aproxima, agravamento significativo, após período de estabilidade e melhoras e quando a mãe é afastada, a criança apresenta melhoras sem novas recidivas (Algeri et al., 2014). A propósito, Rosenberg efetuou um estudo com cerca de 117 casos onde foi possível traçar o perfil da vítima e do agressor (Ferrão \& Neves, 2013), resultando 


\section{NA TEIA DA FICÇÃO: A COMPLEXA NARRATIVA DA SÍNDROME DE MUNCHAUSEN POR PROCURAÇÃo}

num artigo publicado em 1987, sendo que a literatura atual mantém semelhanças com o padrão encontrado na época, designadamente, os casos sendo na sua maioria praticados pela mãe; esta sendo gentil e transmitindo a ideia de cuidadora ao estar todo o tempo junto do filho (designada como relação de fuso); quando confrontada com a prática do abuso, nega perentoriamente; apresenta terapêutica psicológica prévia; manipulação; gosto particular por procedimentos complexos mesmo que arriscados; a preocupação não se revela congruente com a alegada doença; historial clínico extenso e de várias entidades hospitalares; doença com difícil trato; a vítima apresenta reincidências ou o tratamento não se revela eficaz; os exames de diagnóstico não se coadunam com o estado da criança (Ferrão \& Neves, 2013).

Para que a Síndrome de Munchausen por Procuração suceda (Telles et al., 2015), é necessário envolver três entidades, nomeadamente, mãe, filho/a e médico, sendo esta relação instituída de forma triangular (Silva \& Priszkulnik, 2013; Telles et al., 2015). 0 médico é utilizado pelo portador da patologia para perpetuar o abuso, na estreita forma de submeter a criança, "mesmo que de forma inadvertida" (Telles et al., 2015, p. 40) a procedimentos invasivos e muitas vezes dolorosos, com vista à correta categorização nosológica e posterior tratamento, contrariando 0 juramento de Hipócrates (Silva \& Priszkulnik, 2013).

\section{MOTIVOS ASSOCIADOS}

Visando os motivos, de forma genérica, vários autores apontam a "simpatia, atenção, respeito e o facto de ser reconhecida como cuidadora exemplar" (Criddle, 2010, p.50), como motivos primordiais, pois os portadores desta síndrome percecionam-se como importantes quando salvam a criança, o que contribui para elevar a sua autoestima e colmatar a necessidade de estima e autorrealização. Ancorado a estes motivos encontra-se a necessidade de serem reconhecidos, de adquirir e desempenhar um papel, quanto mais não seja junto da "família" hospitalar (Criddle, 2010, p.50). "Muitas teorias existem para explicar por que essas mães podem fabricar doenças em seus filhos. A mais comum é a perda precoce de suas mães, que é um dado frequente nos casos de SMPP. Essa perda representa uma rejeição e uma falta de amor e atenção na infância" (Pires \& Molle, 1999, p. 284), 0 que pode sugerir a necessidade intrínseca de obter atenção. Saliente-se que alguns estudos correlacionam maus tratos na infância com os portadores da Síndrome de Munchausen por Procuração (Telles et al., 2015). Contudo a literatura não se mostra unânime sobre o tema.

\section{CONCLUSÃO}

A narrativa da Síndrome de Munchausen por Procuração, em virtude das suas características, assume-se como uma tipologia de mau trato infantil que pode culminar na morte da criança, estando esta em perigo.

Dada a natureza da sua condição, também se assume de difícil diagnóstico e perceção atempada pelos diversos profissionais. A sua não identificação sustenta as necessidades da mãe e os motivos que lhe estão associados, podendo conduzir o profissional de saúde a quebrar o juramento de Hipócrates pela perpetuação do ciclo abusivo, pois este transtorno necessita de três elementos, envolvendo assim uma relação triangular, onde o médico assume um papel de elevada importância configurado em dois sentidos, na perpetuação do abuso e no diagnóstico oportuno. É de ressalvar que, em virtude dos motivos apresentados, há correlação com a escalada criminal, significando, portanto, uma continuação na busca incessante da satisfação das necessidades internas vinculadas à patologia e por conseguinte uma escalada de violência, sendo imprescindível a deteção precoce desta conjuntura.

Em suma, é de extrema relevância a capacitação de profissionais das mais diversas áreas e novas investigações no meio académico, visando o fim último do superior interesse da criança.

\section{REFERÊNCIAS BIBLIOGRÁFICAS}

Algeri, S, Silva, F. M., Anflôr, E. P., Oliveira, C. P., \& Costa, A. C. (2015). Síndrome de Munchausen por Procuração: Revisão Integrativa. Revista Enfermagem UFPE on line, 8(11), 3983-91. Disponível em Consultado em Abril 2, 2021, em 
https://www.lume.ufrgs.br/bitstream/handle/10183/107469/000944252.pdf?sequence=1.

American Psychiactric Association. (2013). Somatic Symptom and Related Disorders. In: Diagnostic and Statistical Manual of Mental Disorders, 5th ed.; Arlington, VA: American Psychiactric Association, pp. 309329.

Cascais, M. M. P. (2012). Síndrome de Munchausen por Procuração, um desafio clínico. Dissertação de mestrado, Universidade do Coimbra, Coimbra, Portugal.

Comert, I. T., Ugras, S., Islek, D. S. \& Yukseloglu, H. E. (2018). A review about munchausen syndrome by proxy: form of child abuse. Forensic Research \& Criminology International Journal 6(2), 86-88. Consultado em outubro 13, 2021, em https://medcraveonline.com/FRCIJ/a-review-about-munchausen-syndrome-byproxy-form-of-child-abuse.html.

Criddle, L. (2010). Monsters in the Closet: Munchausen Syndrome by Proxy.Critical Care Nurse, 30(6), 46-55. Consultado em Abril 5, 2021, em https://citeseerx.ist.psu.edu/viewdoc/download?doi=10.1.1.372.239\&rep=rep1\&type=pdf.

Ferrão, A. C. F. \& Neves, M. G. C. (2013). Síndrome de Munchausen por Procuração: quando a mãe adoece 0 filho. Com.Ciências Saúde 24(2), 179-186. Consultado em Abril, 14, 2021 em http://bvsms.saude.gov.br/bvs/artigos/ccs/sindrome_munchausen_procuracao.pdf.

Filho, D. S., Kanomata, E. Y., Feldman, R-. J. \& Neto, A. M. (2017). Síndrome de Munchausen e síndrome de Munchausen por procuração: uma revisão narrativa. Revista Einstein 15(4), 516-21. Consultado em abril 19, 2021 em http://apps.einstein.br/revista/arquivos/PDF/3746-516-521-Sindrome-de-Munchausen-e-sindrome-de-Munchausen-por-procuracao.pdf.

Franco, J. O.B., Batistão, R. S., Santos, D. C., Ferreira, W. F. S. \& Vasconcelos, C. R. (2020). Biotética e sociedade: transtorno factício autoimposto e imposto a outro. Revista LatinoAmerica de Biotética 20(1), 49-66. Consultado em Outubro 11, 2021, em http://www.scielo.org.co/scielo.php?pid=S165747022020000100049\&script=sci_abstract\&tlng=pt.

Greiner, M. V., Palusci, V. J., Keeshin, B. R. \& Kerans, S. C. (2015). A Preliminary Screening Instrument for Early Detection of Medical Child Abuse. Hospital Pediatrics 3(1), 39-44. Consultado em Maio 6, 2021, em https://www.researchgate.net/publication/259250113_A_Preliminary_Screening_Instrument_for_Early_Det ection_of_Medical_Child_Abuse.

Pires, J. M. A. \& Molle, L. D. (1999). Síndrome de Munchausen por procuração - relato de dois casos. Jornal de Pediatria (Rio de Janeiro) 75(4), 281-286. Consultado em Abril 1, 2021, em http://www.jped.com.br/conteudo/99-75-04-281/port.pdf.

Silva, H. M. \& Priszkulnik, L. (2013). Síndrome de Munchausen por procuração, a Psicologia e a Psicanálise: conhecer para suspeitar. Psicologia,Conocimiento y Sociedad 3(2), 155-170. Consultado em Abril 24, 2021 em https://www.redalyc.org/pdf/4758/475847410008.pdf.

Tachibana, M. \& Ferreira, G. D. (2020). 0 cuidado materno violento: reflexões psicanalíticas sobre a Síndrome de Munchausen por Procuração. Semina: Ciências Sociais e Humanas, Londrina 41(2), 229-248. Consultado em Abril 4, 2021 em http://www.uel.br/revistas/uel/index.php/seminasoc/article/viewFile/39019/27978.

Telles, L. E. B., Moreira, C. G., Almeida, M. R. Mecler, K., Valença, A. M. \& Baldez, D. P. (2015). Transtorno Factício Imposto a Outro (Síndrome de Munchausen por Procuração) e Maus-Tratos Infantis. Revista Debates em Psiquiatria, 38-43. Consultado em Maio 5, 2021 em https://www.researchgate.net/profile/Lisieux-Telles2/publication/325321599_ARTIGO_TRANSTORNO_FACTICI0/links/5b059858aca2725783d89454/ARTIGO-TRANSTORNO-FACTICIO.pdf.

World Health Organization. (2021). The ICD-10 Version 2019 - International Statistical Classification of Diseases and Related Health Problems - Classification mental and Behavioural Disorders. [Em linha]. World Health Organization Web site. Consultado em Maio 2, 2021 em https://icd.who.int/browse10/2019/en\#/V.

Zenoni, A. (2002). Quando o filho realiza o objeto (A respeito da chamada "síndrome de Munchausen por procu- 


\section{NA TEIA DA FICÇÃO: A COMPLEXA NARRATIVA DA SÍNDROME DE MUNCHAUSEN POR PROCURAÇÃo}

ração"). Psicologia em Revista 8(12), 65- 71. Consultado em Abril 10, 2021 em

https://www.researchgate.net/publication/277796137_Quando_0_filho_realiza_0_objeto_A_respeito_da_chamada_sindrome_de_Munchausen_por_procuracao. 